\title{
Prevalence, Structure, and Risk Factors for Mental Disorders in Older People
}

\author{
N. N. Petrova ${ }^{a, *}$ and D. A. Khvostikova ${ }^{a}$ \\ a St. Petersburg State University, St. Petersburg, 199106 Russia \\ *e-mail:petrova_nn@mail.ru
}

Received June 30, 2020; revised January 5, 2021; accepted January 11, 2021

\begin{abstract}
This review focuses on assessing the prevalence and risk factors of mental disorders in older people in the modern era, including the COVID-19 pandemic. A systematic review of the literature was conducted in PubMed, Elsevier, and Google using keywords over the past 10 years. Substantial discrepancy of data on the prevalence of psychiatric disorders has been shown in the elderly population. The significant incidence of mental disorders among nursing home residents is highlighted. The relevance of nonpsychotic depressive and anxiety disorders is demonstrated for the elderly, along with the difficulty of diagnosing mental disorders associated with physical pathology and cognitive impairments. The risk factors for mental disorders in older adults are socio-demographic but also economic, psychological, and physical. The problem of mental health of the elderly is characterized for the conditions of the COVID-19 pandemic associated with specific risk factors for psychiatric disorders. The shortage of evidence-based research in the treatment of mental disorders in the elderly and the urgency to improve the organization of psychiatric care for such patients are noted. Understanding the structure and prevalence of mental disorders among the elderly will allow optimizing the functioning of healthcare systems.
\end{abstract}

Keywords: mental disorders, prevalence, structure, risk factors, older age, COVID-19, treatment

DOI: $10.1134 /$ S2079057021040093

\section{INTRODUCTION}

Currently, an increase in the relative and absolute number of elderly people is seen around the world. Between 2015 and 2050 the proportion of the elderly population will rise from 12 to $22 \%$, reaching 2 billion people. Due to the reduction of young mortality, the majority of people from low- and middle-income countries might attain the age of 60 years, whereas in high-income countries the life expectancy is constantly increasing by the reduction of mortality of the elderly. Psychiatric disorders and cognitive impairments are one of the problems interfering with active ageing. Over $20 \%$ of adults older than 60 years suffer from mental or neurological diseases, apart from headache-related ones, causing $6.6 \%$ of total disability in people of this age group [65]. There is a wide span of mental illnesses that vary from mild personality changes to severe disintegration of mental activity; furthermore, an atypical clinical picture of psychiatric disorders is seen in patients of presenile and senile age that is often asymptomatic or with unspecific manifestations, and susceptibility to iatrogenic interventions [4]. Understanding the structure and prevalence of mental disorders will help optimize the functioning of healthcare systems in assisting the elderly population [8].
The purpose of this review was to analyze the studies assessing the incidence and risk factors of psychiatric disorders in the elderly in the modern era, including the COVID-19 pandemic.

A systematic review of the literature was conducted in PubMed, Elsevier, and Google using keywords over the past 10 years.

\section{MENTAL DISORDERS IN OLDER PEOPLE AND THEIR PREVALENCE}

The data regarding the prevalence and structure of mental disorders in old people significantly vary. There is an opinion that depression is a prevailing component in the structure of psychiatric pathology of the elderly, hitting $28.3 \%$ in some countries [23]. The incidence of major depressive disorder reaches $2.8 \%$ for elderly people in China [62]. According to other sources, depressive symptoms of different severity are observed in 10-25\% of people above 65 years old [29], their incidence increasing after the age of 81 [22]. In $5.6 \%$ of cases depression is represented by a severe depressive episode [52]. A recent population study in Korea has discovered that depression (up to $17.8 \%$ ) and anxiety disorders (up to 15\%) are the most common psychiatric diseases [36]. Referring to other authors, anxiety disorders predominate over depres- 
sion, their prevalence being $11.4-12.3 \%$, including $5.8 \%$ of phobic and $6.8-9.1 \%$ of mood disorders [34]. Anxiety and depression are often comorbid, which hampers the diagnosis. Among the elderly people 13\% suffering from anxiety disorder also have depression and $36 \%$ of depressive patients have a concomitant anxiety disorder [65]. At least one criterion of personality disorder can be found in $14.5 \%$ of old people with obsessive-compulsive disorder $(6.5 \%)$ being preponderant.

It is thought that mental disorders are much more common among older people than in the young and middle-aged population. Thus, the WHO reports that 236 old people per 100000 suffer from a psychiatric disorder, as opposed to 93 in the age group of 4564 years. Referring to B. Ausín et al., every second person above 65 years old suffers from a certain mental disease sometime in their lives, every third in the past year, and every fifth, at the moment of observation [12]. However, contradicting data are also present. The study of A. Gum et al., detected mental disease in $8.5 \%$ of people aged above 65 years during their lifetime, $22.4 \%$ for the age of $45-64$ years, and $27.6 \%$ for 18-44 years [27]. This divergence might be associated with long-term memory loss associated with ageing, differences in the diagnosing methods, and premature death of patients with psychiatric diseases.

Due to the increased risk of adverse life events in the presenile age, a high prevalence of depression can be expected. Nevertheless, at the end of the 20th century several researchers have discovered an increased share of affective disorders in the elderly throughout life, particularly, a U-shaped change in the proportion with the maximal levels seen in the young and elderly population and an opposing reduction of in the proportion of affective disorders [41]. It should be emphasized that older people suffer from symptoms of depression not fulfilling the criteria of major depression disorder more often than young adults. Furthermore, subthreshold depression is a risk factor for clinically significant depression, diagnosed in every tenth senior [1].

It is believed that the prevalence of anxiety disorders in the elderly is slightly lower than in young adults [53], their incidence declines with age from $20-30 \%$ in $20-40$-year-old people to $3-5 \%$ in those above 65 years old. These age-related changes in the prevalence of anxiety disorders might be associated with their age-related transformation into other diseases, particularly, depression, dementia, psychoactive substance dependence, and physical disorders. Besides, early mortality is typical for people with anxiety disorders, whereas new cases of anxiety disorders are rare among elderly people. The incompleteness of classification plays a certain role as well, favoring underdiagnosis of anxiety disorders in the older population.

The prevalence of posttraumatic stress disorder of the anxiety spectrum can reach $5.5 \%$ in the elderly people. Typical factors for ageing, such as changes of social roles, retirement, bereavement, physical health conditions decreasing their autonomy, and the lack of social support, might be related to the exacerbation of posttraumatic stress symptoms [33].

The combination of mental and cognitive impairments within neurodegeneration is one of the most common types of comorbid mental disorders among the elderly [28]. The incidence of cognitive impairments varies from $7.6 \%$ in India [59] to $20 \%$ in China [19]. It has been shown that depression is associated with the highest risk of cognitive impairments. According to meta-analysis, a major depression disorder is seen in $15.9 \%$ of patients with dementia, deteriorating the prognosis and increasing the need for constant care and mortality [11].

S. Ducharme et al., conducted a review of the approaches to differential diagnosis of the behavioral variant frontotemporal dementia (FTD, or Pick's disease) and primary mental disorders unrelated to dementia [21]. The group of authors included the leading specialists from different countries (United States, Canada, Australia, Netherlands, Germany, Belgium, France, Italy, etc.), allowing us to accumulate the international experience on this issue. The psychopathological background of behavioral variant FTD can mimic mental disorders. Noncognitive psychiatric diseases can be found in $73 \%$ of patients at the onset of behavioral variant FTD, exacerbating the disease progression, and observed in all patients at the stage of moderate dementia. Eating disorders are diagnosed in $60 \%$ of patients at the onset and in over $80 \%$ of patients at the advanced stage of the disease, whereas mood disorders can be detected in $35-25 \%$ of patients with behavioral variant FTD, often developing long before establishing the diagnosis. Clinical overlap of FTD symptoms with mood, obsessivecompulsive, schizophrenic, and personality disorders at the early stage of disease development underlies common diagnosing errors, when the mental diseases are the primary diagnosis unrelated to neurodegenerative dementia, potentially postponing the initiation of pathogenic treatment for several years. The authors emphasize the value of objective anamnesis and dynamical estimation of disease course (symptom fluctuation) for diagnosing, along with the relevance of an interdisciplinary approach to these patients with obligatory consultations of both neurologists and psychiatrists.

Mental disorders with comorbid physical diseases are another problem for elderly people. A large number of elderly patients suffering from depressive and cognitive impairments are admitted to multidisciplinary hospitals; moreover, concomitant depression, anxiety, and hypochondriacal disorders are difficult to diagnose in patients with multiple physical diseases [5]. Depression is poorly distinguished in people of presenile and senile age in general medical practice. 
A retrospective study of the mental disease structure comprising the patients admitted to family clinic no. 9 of Toshkent aged over 55 years discovered the preponderance of diseases associated with brain damage and dysfunction of organic nature $(44.4 \%)$, response to severe stress and maladaptation $(29.5 \%)$, behavior and personality disorders related to brain disease, damage, or dysfunction (16.7\%), and depressive episodes (5.6\%). Patients often reported sleep impairment (85.2\%), persistent dysphoria $(81.5 \%)$, and anxiety $(61 \%)$. Delusions of influence or detriment were seen in $27.8 \%$ of patients (33.3\% each) [6].

The frequency of mental disorders is substantial among elderly nursing home residents. For instance, over 4.7 million of the United States population stays in nursing homes, $80 \%$ of them older than 65 years and $40 \%$ of them suffer from mental diseases. The prevalence of depression and anxiety disorders is 28 and $18.9 \%$, respectively [63]. According to researchers from Iraq, the share of presenile people with mental disorders is $55.8 \%$ for nursing home residents and $21.5 \%$ of those living with their families; depression, anxiety, and dementia being the most prevalent [31]. It has been noted that the incidence of depression in the elderly in long-term care homes is around $10 \%$, which is higher than in the population [54]. Mental disorders in home care elders have a more severe course than in the general population and depression is more frequently accompanied by suicidal ideations [63]. This can ensue from the development of physical illness, severe functional disturbances, interfering with independent living, and exposure to such stress factors as loss of home, spouse, social contacts, and loneliness [70].

\section{THE RISK FACTORS FOR MENTAL DISORDERS IN THE ELDERLY}

The risk factors for mental disorders in the presenile population include being female, loneliness, alcohol abuse, lack of education, financial shortage, a family history of mental diseases, and severe physical disease [2, 14, 57, 67, 69].

K. Reynolds et al. [52], have analyzed the mental condition of over 12000 people older than 55 years in the United States. Statistical differences were registered in dependence from sex, ethnicity, relationship, and economical status. The proportion of mood and anxiety disorders was higher in females, whereas in males, psychoactive substance dependence and personality disorders were preponderant [12]. It should be emphasized that sex-related differences in mental disorders depend on country and other social contexts. The factors that can help in interpreting these differences comprise of cultural and social norms, as well as the variations of gender roles and coping strategies between men and women [35]. Domestic violence is closely related to mental health issues in the elderly. Around $20-30 \%$ of females after turning 65 experience family abuse in their lifetime, and while the pro- portion of physical violence decreases with age, the emotional abuse remains steady throughout life [37].

The results of several studies confirmed the increase of borderline disorder morbidity rate in presenile and senile age, including reactive depression, majorly influenced by stressful events related to loneliness, uselessness, and loss of life purpose. It was demonstrated that satisfaction with life, i.e., the level of happiness, in old age was affected by such factors as relationship status, family income, and social activity [46]. These results coincide with the data of Chinese scientists, suggesting that sufficient social support promotes social activity and mental health of the elderly [16]. Economic and social crises as an example of social factor influence can have a negative impact on psychological conditions, hence the higher prevalence of mental diseases in the elderly people of Iraq throughout their lifetime compared to Europe (38.7\% [31] against $23.3 \%$ [8]).

The region of living also contributes to the risk of mental disorder development. Several studies indicate the predominance of mental illnesses among citizens in cities compared to rural populations $[51,55]$. This is probably related to the pace of life; thus, it has been shown that the risk of psychiatric diseases is lower in small towns with a peaceful rhythm of life. Continuous urbanization together with population ageing leads to the growing incidence of mental disorders in the elderly and enhancement of the burden on the healthcare system [23, 44]. Income and relationship status are also crucial risk factors for the development of mental diseases in citizens [43, 64].

However, the example of China demonstrates that mental health issues are more commonly seen in presenile people inhabiting rural areas [32]. The share of mental disorders can reach up to $20.11 \%$ in elderly people living in the countryside, while the prevalence of severe depression is $9.2 \%$ [19]. The risk factors for mental disorders in countrymen include low income, chronic physical illnesses, and migration of children to big cities, especially of an only child, resulting in the feeling of loneliness, limitation of social interactions, and lack of social support. The combination of these factors substantiates a more severe course of mental disorders in the rural area $[9,17]$. In general, the governmental social support is worse in the countryside than in the cities and old people are often financially dependent on children [30]. It is assumed that the relationship status is the major predictor of depression in senior age. Lonely, divorced, and widowed elderly people often experience loneliness, lack of social support, and insecurity, thus increasing the risk of depression. Young people in developing poor countries looking for jobs often have to leave their presenile parents. Mexico is an example demonstrating that the absence of contact with children leads to the development of loneliness and negative emotional sufferings of presenile people, which in turn provokes psychiatric distur- 
bances. Illegally staying in another country as irregular migrants and their families complicates contact with elderly parents and promotes the establishment of psychological distress [20]. Nevertheless, many old people are able to psychologically cope with the migration of their children, as shown in the Moldavian population, positively looking at their financial wellbeing and helping their children and grandchildren [15].

There is a strong correlation between mental and physical health, associated with both direct reciprocal influence and indirect effects. Indirect effects explain the influence of physical health on the psychological one in $8 \%$ of cases, whereas in $10 \%$ of cases this influence is reversed. The previous psychological health has a stronger impact on the actual physical health than physical exercise or education. On the contrary, the state of physical wellbeing in the past has a larger contribution to the present psychological condition than health investments, income, or education [47].

The development of depression can result from physical diseases, including endocrine diseases, organic brain lesions due to vascular pathology, or age-specific diseases such as Parkinson or Alzheimer disease, and administration of certain drugs. The combination of depression and obesity is typical for older people, which is possibly related to the disturbance of physical functioning. Body weight loss with the help of diet or surgical intervention leads to the amelioration of depressive symptoms, reduces stress levels, and improves the quality of life. Furthermore, mental disorders serve as predictors of poor response to weight loss interventions [49]. Sufficient physical activity and a balanced diet full of antioxidants, omega-3 fatty acids, and B vitamins positively affect mental health $[50,60]$. The administration of calcium and $\mathrm{D}$ vitamin drugs reduces the risk of depressive symptoms in the elderly [66].

\section{MENTAL HEALTH IN ELDERLY PEOPLE AND COVID-19}

The mental health of people all over the world was adversely affected by direct and indirect economic damages and psychosocial distress together with significant restriction of access to medical care for people with health issues caused by the COVID-19 pandemic and measures to combat it. Social distancing and selfisolation only add to stress. The significance of social interaction for mental health of older people showed itself especially prominently during the COVID-19 pandemic, particularly, stay-at-home orders and personal distancing promoted an outbreak of depression among the elderly, specifically those living alone without children [40].

Social distancing or self-isolation and loneliness, along with the restriction on freedom of movement and physical exercising can be highly dangerous for mental health and spiritual comfort of adolescents and elders compared to young adults. Isolation is associated with a sedentary life, which especially adversely affects the elderly. The lack of exercising causes functional disturbances and permanent need for physical assistance, which in turn poorly influence emotional and psychological wellbeing [24]. Besides, economic stagnation and deterioration of the financial state are additional sources of stress [7, 38, 61]. The novel unknown situation plays a role of stressor, aggravating the mental state of old people. Thus, in the United States the share of people older than 65 years who reported negative consequences of COVID-19 pandemic for psychological health to the interviewers increased from 27 to 47\% during March and July [48]. Quarantine led to the elevation of cases of posttraumatic stress disorder and increase in anxiety and irritation levels of old people [18].

Social distancing, anxiety, fear of contamination, uncertainty, chronic stress, and economic difficulties can provoke the development or exacerbation of depression, anxiety, and psychoactive substance dependence, which in turn increases the risk of suicide. Social isolation and feeling of loneliness themselves are associated with suicidal ideations and behavior. The ability to have contact with friends and relatives using electronic devices, healthy sleep, food, and exercising help to reduce the risk of suicide. Presenile people are exposed to an elevated risk of suicide, especially with an already existing mental disorder. It is recommended to limit watching the TV shows potentially referring to unreliable sources, thus enhancing psychoemotional distress [56].

Public healthcare professionals tend to estimate subtle changes in mental health of the elderly, since the duration of isolation remains undetermined [13]. The improvement of telemedicine and access to helplines might decrease psychoemotional distress among the elderly [26]. However, it should not be forgotten that using Internet sources and smartphones are especially challenging for the majority of old people [68].

\section{CARE ORGANIZATION}

Several factors are considered pivotal for the positive result of medical interventions in mental disorders of elderly people, motivation being the key component. If presenile people are not interested in treatment results, it will not be effective enough. Thus, personal preferences and demands of the elderly should be taken into account. The interventions should be adjusted depending on the level of physical, cognitive, social, and psychological functioning of the elderly. Group interventions and interventions involving social elements have a strong positive influence on the mental health of elders. Probably, this is due to the fact that many old people face social problems and restriction of social interactions, since the death of relatives and friends is an inevitable part of ageing [45]. 
The situations requiring emergency psychiatric help occur more often among the elderly [10]. Psychotherapy can be beneficial in managing anxiety, depression, and posttraumatic stress disorder, though its efficiency for comorbid old people is unclear. Social impacts positively affect the cognitive functions of patients. Currently, the possibility is considered to help old patients over the phone and engage volunteers, using cognitive behavioral therapy [42]. There is evidence that selective serotonin reuptake inhibitors are efficient in treating mood disorders [58].

Pharmacological treatment of old patients requires special vigilance because of the increased risk of drug interactions and side effects [39]. Therefore, comorbid elderly patients are often prescribed low drug doses to avoid adverse effects, which complicates the evaluation of actual efficiency and safety of individual drugs and their combinations in geropsychiatric practice. V.G. Rotshtein et al., discovered that patient contingents differ by clinical and sociodemographic characteristics between psychiatric facilities, introducing two different treatment goals, ranging from full recovery of a patient's social opportunities to maintenance on a level satisfied by outpatient treatment only [3]. As a result, each of the investigated types of facilities elaborated their own arsenal of drugs that could more or less allow achieving the therapeutic goals, suitable for each of the mentioned contingents of involutional and presenile age. A special safety analysis for psychotropic drugs should be conducted for elderly patients.

Only around $20 \%$ of patients with mental disorders seek professional help [34]. Late diagnosis of mental diseases in elderly patients aggravates the disease burden for their microsocial environment, healthcare system, and society in general $[25,44]$.

\section{CONCLUSIONS}

Because of the growing number of elderly people in the population, the international experts expect the increase in prevalence of mental diseases among them, therefore the improvement of diagnosis of mental health issues and mental health system gains importance in the older age group in accordance with national, historical characteristics, and socioeconomic living conditions. The significance of socioeconomic factors in the development of psychiatric diseases of the elderly is rising in modern society. Nonpsychotic mental diseases of mood or anxiety spectrum become more challenging along with comorbid mental, physical, and cognitive impairments. There are substantial problems with standardizing the approaches to diagnosis and treatment of mental diseases in old people. The organization of geropsychiatric help becomes the task of prime importance in modern psychiatry due to the incidence of mental disorders, high prevalence of comorbid pathologies, and difficulties in treating old age groups.

\section{COMPLIANCE WITH ETHICAL STANDARDS}

Conflict of interests. The authors declare that there is no conflict of interest.

Statement of compliance with standards of research involving humans as subjects. All procedures performed in studies involving human participants were in accordance with the ethical standards of the institutional and/or national research committee and with the 1964 Helsinki Declaration and its later amendments or comparable ethical standards. Informed consent was obtained from all individual participants involved in the study.

\section{REFERENCES}

1. Vishnyakov, Yu.V. and Desyatninenko, I.V., Treatment and rehabilitation of depressive disorders in patients of a geriatric boarding school, Arkh. Vnutr. Med., 2016, vol. 6 , suppl. 1, pp. 86-87.

2. Guzova, A.V., Golubeva, E.Yu., and Solov'ev, A.G., Quality of life and biological age of elderly people with alcohol dependence in the conditions of European North, Usp. Gerontol., 2010, vol. 23, no. 1, pp. 110-114.

3. Rotshtein, V.G., Safarova, T.P., Sheshenin, V.S., et al., Evaluation of psychopharmacotherapy in elderly patients in different psychiatric settings, $Z$ h. Nevropatol. Psikhiatr. im. S.S. Korsakova, 2013, vol. 113, no. 2, pp. 59-65.

4. Solov'ev, A.G., Golubeva, E.Yu., and Pezeshkian, Kh., The role of the social environment in screening for mental health disorders in the elderly people in the near-Arctic zone of Russia, Ekol. Chel., 2019, no. 2, pp. 60-64.

5. Fedotov, D.D., Sheshenin, V.S., Polishchuk, Yu.I., and Varentsova, V.A., Mental disorders in elderly patients treated in a multidisciplinary hospital, Med. Ekstremal'nykh Situatsii, 2016, vol. 2, no. 56, pp. 37-44.

6. Kholtaeva, F.F. and Alimova, G.U., Mental disorders in elderly and senile persons, Arkh. Vnutr. Med., 2016, special issue, pp. 85-86.

7. Ahorsu, D.K., Lin, C.Y., and Pakpour, A.H., The association between health status and insomnia, mental health, and preventive behaviors: the mediating role of fear of COVID-19, Gerontol. Geriatr. Med., 2020, vol. 6 . https://doi.org/10.1177/2333721420966081

8. Andreas, S., Schulz, H., Volkert, J., et al., Prevalence of mental disorders in elderly people: the European MentDis_ICF65+ study, Br. J. Psychiatry, 2017, vol. 210, no. 2, pp. 125-131.

9. Ao, X., Jiang, D., and Zhao, Z., The impact of ruralurban migration on the health of the left-behind parents, China Econ. Rev., 2016, vol. 37, pp. 126-139.

10. Arguvanli, S., Akin, S., Deniz Safak, E., et al., Prevalence of cognitive impairment and related risk factors in community-dwelling elderly in Kayseri, Turkey, Turk. J. Med. Sci., 2015, vol. 45, no. 5, pp. 1167-1172.

11. Asmer, S., Kirkham, J., Newton, H., et al., Meta-analysis of the prevalence of major depressive disorder among older adults with dementia, J. Clin. Psychiatry, 2018 , vol. 79 , no. 5 .

https://doi.org/10.4088/JCP.17r11772 
12. Ausín, B., Muñoz, M., Santos-Olmo, A., et al., Prevalence of mental disorders in the elderly in the community of Madrid: results of the Mentdis_ICF65+ study, Span. J. Psychol., 2017, vol. 20, pp. 1- 11 .

13. Baker, E. and Clark, L.L., Biopsychopharmacosocial approach to assess impact of social distancing and isolation on mental health in older adults, Br. J. Commun. Nurs., 2020, vol. 25, no. 5, pp. 231-238.

14. Baladón, L., Fernández, A., Rubio-Valera, M., et al., Prevalence of mental disorders in non-demented elderly people in primary care, Int. Psychogeriat., 2015, vol. 27, no. 5, pp. 757-768.

15. Böhme, M.H., Persian, R., and Stöhr, T., Alone but better off? Adult child migration and health of elderly parents in Moldova, J. Health Econ., 2015, vol. 39, pp. 211-227.

16. Chao, S.-F. and Chen, Y.-C., Environment patterns and mental health of older adults in long-term care facilities: the role of activity profiles, Aging Ment. Health, 2019, vol. 23, no. 10, pp. 1307-1316.

17. Chen, C.-M., Lee, I.-C., Su, Y.-Y., et al., The longitudinal relationship between mental health disorders and chronic disease for older adults: a population-based study, Int. J. Geriatr. Psychiatry, 2017, vol. 32, no. 9, pp. 1017-1026.

18. Chong, T., Curran, E., Ames, D., et al., Mental health of older adults during the COVID-19 pandemic: lessons from history to guide our future, Int. Psychogeriatr., 2020. https://doi.org/10.1017/S1041610220001003

19. Ding, Z., Wang, G., Zhang, Y., et al., Epidemiological survey of mental disorders in the rural left behind elderly aged 60 years and older in Gansu, Zhonghua Yi Xue Za Zhi, 2019, vol. 99, no. 31, pp. 2429-2434.

20. Downer, B., González-González, C., Goldman, N., et al., The effect of adult children living in the United States on the likelihood of cognitive impairment for older parents living in Mexico, Ethnicity Health, 2018, vol. 23 , no. 1 , pp. 57-71.

21. Ducharme, S., Dols, A., Laforce, R., et al., Recommendations to distinguish behavioural variant frontotemporal dementia from psychiatric disorders, Brain, 2020, vol. 143, no. 6, pp. 1632-1650.

22. Forlani, C., Morri, M., Ferrari, B., et al., Prevalence and gender differences in late-life depression: a population-based study, Am. J. Geriatr. Psychiatry, 2014, vol. 22, no. 4, pp. 370-380.

23. Giri, M., Chen, T., Yu, W., and Lü, Y., Prevalence and correlates of cognitive impairment and depression among elderly people in the world's fastest growing city, Chongqing, People's Republic of China, Clin. Interventions Aging, 2016, vol. 11, pp. 1091-1098.

24. Goethals, L., Barth, N., Guyot, J., et al., Impact of home quarantine on physical activity among older adults living at home during the COVID-19 pandemic: qualitative interview study, JMIR Aging, 2020, vol. 3, no. 1. https://doi.org/10.2196/19007

25. Gontijo Guerra, S., Berbiche, D., and Vasiliadis, H.M., Changes in instrumental activities of daily living functioning associated with concurrent common mental disorders and physical multimorbidity in older adults, Disabil. Rehabil., 2020.

https://doi.org/10.1080/09638288.2020.1745303
26. Goodman-Casanova, J.M., Dura-Perez, E., GuzmanParra, J., et al., Telehealth home support during COVID-19 confinement for community-dwelling older adults with mild cognitive impairment or mild dementia: survey study, J. Med. Internet Res., 2020, vol. 22, no. 5. https://doi.org/10.2196/19434

27. Gum, A., King-Kallimanis, B., and Kohn, R., Prevalence of mood, anxiety, and substance-abuse disorders for older Americans in the National Comorbidity Survey-replication, Am. J. Geriatr. Psychiatry, 2009, vol. 17, no. 9, pp. 769-781.

28. Guo, Y.-Q., Zhang, C.-C., Huang, H., et al., Mental health and related influencing factors among the empty-nest elderly and the non-empty-nest elderly in Taiyuan, China: a cross-sectional study, Publ. Health, 2016, vol. 141, pp. 210-217.

29. Heser, K., Tebarth, F., Wiese, B., et al., Age of major depression onset, depressive symptoms, and risk for subsequent dementia: results of the German study on Ageing, Cognition, and Dementia in Primary Care Patients (AgeCoDe), Psychol. Med., 2013, vol. 43, no. 8, pp. 1597-1610.

30. Hu, H., Cao, Q., Shi, Z., et al., Social support and depressive symptom disparity between urban and rural older adults in China, J. Affective Disord., 2018, vol. 237, pp. 104-111.

31. Ibrahim, A., AI-Lami, F., Al-Rudainy, R., and Khader, Y., Mental disorders among the elderly people in Baghdad, Iraq, 2017, J. Health Care Org., Provis, Financ., 2019, vol. 56, pp. 1-8.

32. Jin, Y., Zhang, Y.-S., Zhang, Q., et al., Prevalence and socio-demographic correlates of poor mental health among older adults in agricultural areas of China, Front. Psychiatry, 2020, vol. 11. https://doi.org/10.3389/fpsyt.2020.549148

33. Kaiser, A., Cook, J., Glick, D., et al., Posttraumatic stress disorder in older adults: a conceptual review, Clin. Gerontol., 2019, vol. 42, no. 4, pp. 359-376.

34. Karam, G., Itani, L., Fayyad, J., et al., Prevalence, correlates, and treatment of mental disorders among Lebanese older adults: a national study, Am. J. Geriatr. Psychiatry, 2016, vol. 24, no. 4, pp. 278-286.

35. Kiely, K.M., Brady, B., and Byles, J., Gender, mental health and ageing, Maturitas, 2019, vol. 129, pp. 76-84.

36. Kim, C. and Ko, H., The impact of self-compassion on mental health, sleep, quality of life and life satisfaction among older adults, Geriatr. Nurs., 2018, vol. 39, pp. 623-628.

37. Knight, L. and Hester, M., Domestic violence and mental health in older adults, Int. Rev. Psychiatry, 2016, vol. 28, no. 5, pp. 464-474.

38. Le, H.T., Lai, A.X., Sun, J., et al., Anxiety and depression among people under the nationwide partial lockdown in Vietnam, Front. Publ. Health, 2020, vol. 8. https://doi.org/10.3389/fpubh.2020.589359

39. Marin, M., Maftum, M., and Lacerda, M., Elderly people with mental disorders: experiencing the use of psychotropic medicines, Rev. Bras. Enferm., 2018, vol. 71, no. 2, pp. 835-843.

40. Marroquín, B., Vine, V., and Morgan, R., Mental health during the COVID-19 pandemic: effects of stayat-home policies, social distancing behavior, and social resources, Psychiatry Res., 2020, vol. 293. https://doi.org/10.1016/j.psychres.2020.113419 
41. Mirowsky, J. and Reynolds, J., Age, depression, and attrition in the National Survey of Families and Households, Sociol. Methods Res., 2000, vol. 28, no. 4, pp. $476-504$.

42. Moye, J., Innovating mental health treatment for older adults, Clin. Gerontol., 2019, vol. 42, no. 4, pp. 345-346.

43. Nayak, S., Mohapatra, M.K., and Panda, B., Prevalence of and factors contributing to anxiety, depression and cognitive disorders among urban elderly in Odisha-a study through the health systems' Lens, Arch. Gerontol. Geriatr., 2019, vol. 80, pp. 38-45.

44. Neumann, L., Dapp, U., Jacobsen, W., et al., The MINDMAP project: mental well-being in urban environments, Z. Gerontol. Geriatr., 2017, vol. 50, no. 7, pp. $588-602$.

45. Niclasen, J., Lund, L., Obel, C., et al., Mental health interventions among older adults: a systematic review, Scand. J. Publ. Health, 2019, vol. 47, no. 2, pp. 240-250.

46. Nikolakakis, N., Dragioti, E., Paritsis, N., et al., Association between happiness and psychopathology in an elderly regional rural population in Crete, Psychiatriki, 2019, vol. 30, pp. 299-310.

47. Ohrnberger, J., Fichera, E., and Sutton, M., The dynamics of physical and mental health in the older population, J. Econ. Ageing, 2017, vol. 9, pp. 52-62.

48. Panchal, N., Kamal, R., Orgera, K., et al., The implications of COVID-19 for mental health and substance use. https://www.kff.org/coronavirus-covid-19/issuebrief/the-implications-of-covid-19-for-mental-healthand-substance-use/view/footnotes/. Cited November 18, 2020.

49. Payne, M.E., Porter Starr, K.N., Orenduff, M., et al., Quality of life and mental health in older adults with obesity and frailty: associations with a weight loss intervention, J. Nutr. Health Aging, 2018, vol. 22, no. 10, pp. 1259-1265.

50. Pilania, M., Bairwa, M., Khurana, H., et al., Prevalence and predictors of depression in communitydwelling elderly in rural Haryana, India, Indian J. Commun. Med., 2017, vol. 42, no. 1, p. 13

51. Ramaprasad, D., Suryanarayana, R.N., and Kalyanasundaram, S., Disability and quality of life among elderly persons with mental illness, Asian J. Psychiatry, 2015, vol. 18, pp. 31-36.

52. Reynolds, K., Pietrzak, R., El-Gabalawy, R., et al., Prevalence of psychiatric disorders in U.S. older adults: findings from a nationally representative survey, World Psychiatry, 2015, vol. 14, no. 4, pp. 74-81.

53. Schuurmans, J. and van Balkom, A., Late-life anxiety disorders: a review, Curr. Psychiatry Rep., 2011, vol. 13, no. 4, pp. 267-273.

54. Seitz, D., Purandare, N., and Conn, D., Prevalence of psychiatric disorders among older adults in long-term care homes: a systematic review, Int. Psychogeriatr., 2010, vol. 22, no. 7, pp. 1025-1039.

55. Sengupta, P. and Benjamin, A., Prevalence of depression and associated risk factors among the elderly in urban and rural field practice areas of a tertiary care institution in Ludhiana, Indian J. Publ. Health, 2015, vol. 59 , no. 1 , p. 3 .
56. Sher, L., The impact of the COVID-19 pandemic on suicide rates, QJM, 2020, vol. 113, no. 10, pp. 707-712.

57. Stahl, S., Reynolds, C., Whyte, E., and Albert, S., Psychiatric diagnoses among older recipients of publicly funded mental health services, J. Am. Geriatr. Soc., 2017, vol. 65, no. 7, pp. 1569-1572.

58. Tedeschini, E., Levkovitz, Y., Iovieno, N., et al., Efficacy of antidepressants for late-life depression: a metaanalysis and meta-regression of placebo-controlled randomized trials, J. Clin. Psychiatry, 2011, vol. 72, no. 12 , pp. $1660-1668$.

59. Tiwari, S., Tripathi, R., Kumar, A., et al., Prevalence of psychiatric morbidity among urban elderlies: Lucknow elderly study, Indian J. Psychiatry, 2014, vol. 56, no. 2, pp. 154-160.

60. Vecchioli, S.F., Sacchetti, S., Robilant, V.N., et al., The role of physical exercise and omega-3 fatty acids in depressive illness in the elderly, Curr. Neuropharmacol., 2018, vol. 16, no. 3, pp. 308-326.

61. Wadoo, O., Latoo, J., Reagu, S.M., et al., Mental health during COVID-19 in Qatar, Gen. Psychiatry, 2020, vol. 33, no. 6 . https://doi.org/10.1136/gpsych-2020-100313

62. Wang, F., Zhang, Q.-E., Zhang, L., et al., Prevalence of major depressive disorder in older adults in China: a systematic review and meta-analysis, J. Affective Disord., 2018, vol. 241, pp. 297-304.

63. Wang, J., Shang, J., and Kearney, J.A., Mental health disorders in home care elders: an integrative review, Geriatr. Nurs., 2016, vol. 37, no. 1, pp. 44-60.

64. Wang, Z., Shu, D., Dong, B., et al., Anxiety disorders and its risk factors among the Sichuan empty-nest older adults: a cross-sectional study, Arch. Gerontol. Geriatr., 2013, vol. 56, no. 2, pp. 298-302.

65. World report on ageing and health, 2015. https://www.who.int/ageing/events/world-report-2015launch/en/. Cited December 17, 2019.

66. Wu, M.N., He, F., Tang, Q.R., et al., Association between depressive symptoms and supplemental intake of calcium and vitamin D in older adults, J. Nutr. Health Aging, 2020, vol. 24, no. 1, pp. 107-112.

67. Xu, G., Chen, G., Zhou, Q., et al., Prevalence of mental disorders among older Chinese people in Tianjin city, Can. J. Psychiatry, 2017, vol. 62, no. 11, pp. 778786.

68. Yang, Y., Li, W., Zhang, Q., et al., Mental health services for older adults in China during the COVID-19 outbreak, Lancet Psychiatry, 2020, vol. 7, no. 4. https://doi.org/10.1016/S2215-0366(20)30079-1

69. Zhai, Y., Yi, H., Shen, W., et al., Association of empty nest with depressive symptom in a Chinese elderly population: a cross-sectional study, J. Affect Disord., 2015, vol. 187, pp. 218-223.

70. Zhang, C., Hou, L., Zheng, X., et al., Risk factors of mental disorders among empty and non-empty nesters in Shanxi, China: a cross-sectional study, Health Qual. Life Outcomes, 2019, vol. 17. https://doi.org/10.1186/s12955-019-1088-y

Translated by E. Sherstyuk 